\title{
Entretien avec Pierre Ouellet
}

\author{
Entrevista com Pierre Ouellet
}

\author{
Ana Maria Lisboa de Mello \\ Pontifícia Universidade Católica do Rio Grande do Sul - Porto Alegre - Rio Grande do Sul - Brasil \\ Marie-Hélène Paret Passos \\ Pontifícia Universidade Católica do Rio Grande do Sul - Porto Alegre - Rio Grande do Sul - Brasil
}

Zilá Bernd

Universidade Federal do Rio Grande do Sul - Porto Alegre - Rio Grande do Sul - Brasil

Centro Universitário La Salle - Canoas - Rio Grande do Sul - Brasil

\begin{abstract}
Résumé: Pierre Ouellet, né au Québec en 1950, est poète, romancier, essayiste, professeur au département d'Etudes Littéraires de l'Université du Québec à Montréal et titulaire de la Chaire de recherche du Canada en esthétique et poétique. Ses rech'erches se situent dans le champ des relations entre perception et identité dans le discours esthétique, autour de thématiques telles que la littérature migrante, la communauté de mémoire ou encore la voix. Il est membre de l'Académie des Lettres du Québec depuis 2009, directeur de la revue Les Écrits et de la collection Soi et l'autre chez VLB éditeur. Il a publié plus de 40 livres et de très nombreux articles scientifiques, a reçu divers prix dont le prestigieux Prix Ringuet attribué par l'Académie des Lettres du Québec pour Légende dorée, en 1998, et, en 2005 et 2008 le Prix du Gouverneur général, pour les essais $A$ force de voir et Hors-temps. Auteur invité de la $60^{a}$ Feira do livro de Porto Alegre, qui rendait hommage au Canada, Pierre Ouellet a accordé un entretien aux trois professeures: Ana Maria Lisboa de Mello (PUCRS), Marie-Hélène Paret Passos (PUCRS) e Zilá Bernd (Unilasalle Canoas).
\end{abstract}

Mots-clés: Littérature migrante; Communauté de mémoire; Voix; Identité; Québec

Resumo: Pierre Ouellet, nascido em 1950 no Québec, é poeta, romancista, ensaísta, professor titular no departamento de Estudos Literários na Universidade do Québec em Montreal e titular da Cátedra de pesquisa do Canada em estética e poética. Ele trabalha no campo das relações entre percepção e identidade nos discursos estéticos. É membro da Academia de Letras do Québec desde 2009, diretor da revista Les Écrits e da coleção Soi et l'autre de VLB éditeur. Sua obra conta mais de 40 livros publicados e inúmeros artigos científicos. Ele recebeu numerosos prêmios, entre eles o prestigioso Prix Ringuet de l'Académie des lettres du Québec por Légende dorée (1998), e, em 2005 e 2008 o Prix du Gouverneur général, pelos ensaios À force de voir e Hors-temps. Autor convidado da $60^{\mathrm{a}}$ Feira do Livro de Porto Alegre, que homenageava o Canada, Pierre Ouellet concedeu uma entrevista às três professoras: Ana Maria Lisboa de Mello (PUCRS), Marie-Hélène Paret Passos (PUCRS) e Zilá Bern (Unilasalle Canoas).

Palavras-chave: Literatura migrante; Comunidade de memória; Voz; Identidade; Québec

Zilá Bernd: C'est avec une très grande satisfaction que nous vous accueillons ici, à la Pontifícia Universidade Católica do Rio Grande do Sul pour un entretien. Vous venez souvent au Brésil et je sais que vous vous intéressez à la littérature brésilienne. Avec quels auteurs brésiliens vous êtes-vous trouvé des affinités? Peut-être les frères Augusto et Haroldo de Campos, dont je vous ai déjà entendu parler?
Pierre Ouellet: Oui, c'est vrai que c'est la poésie d'abord qui m'a fasciné dans l'espace culturel brésilien. J'avais découvert Oswaldo de Andrade avec le Manifeste anthropophage, il y a déjà plusieurs années, et j'y avais vu des analogies avec les avant-gardes littéraires du Québec dès les années vingt. Il y a une revue qui a été créée au milieu des années 1910 qui s'appelait le Nigog qui est un mot désignant le harpon chez les esquimaux -, 
et l'intérêt que ces poètes d'avant-garde manifestaient pour ce que j'appellerais en gros le primitivisme, ce que l'on a appelé un peu aussi l'indigénisme, c'est-à-dire le retour à des traditions ancestrales extra européennes, à des mythologies qui étaient beaucoup plus, comment dire, amples que celles qui découlaient de la tradition grécoromaine ou judéo-chrétienne. Donc, on est allé dans les avant-gardes du début du siècle, au Québec tout comme au Brésil, on est allé cannibaliser, ni plus ni moins, à travers cette espèce d'anthropophagie culturelle, les mythes, les rituels, les contes, les légendes hérités des premières nations, c'est-à-dire des Inuits et des Innus. Alors, ça m'a fasciné de voir comment de jeunes auteurs, tant au Brésil qu'au Québec, cherchaient une nouvelle langue. JeanAubert Loranger, l'auteur qui a fondé le Nigog, parlait d'une lingua nova, comme une terra nova, une nouvelle terre, nouvelle langue. Et, pour ce faire, c'est-à-dire pour imaginer l'avenir, ces auteurs ont puisé dans un passé très lointain, dans le temps ancestral. On pourrait dire le temps d'avant l'histoire.

ZB: Exactement comme le Manifeste anthropophage.

PO: Tout à fait. Alors, j'ai eu une grande fascination, tout de suite, pour ce type de parole qui a, à la fois, un contenu ethnographique, puisqu'on s'est intéressé visiblement, véritablement à ces cultures, et puis, un caractère, je dirais presque, oui, expérimental de la langue, essayer d'inventer quelque chose comme une langue du nouveau monde. Voilà, c'est pour le Québec comme pour le Brésil, je pense qu'il y a eu cette volonté de se détacher du seul héritage européen, pour adopter d'autres legs, d'autres dons qui venaient d'autres cultures, celles qu'on découvrait sur ce continent, que ce soit au sud ou au nord. Alors, c'est cette parenté qui m'a intéressé tout de suite.

ZB: Il y a, en fait, le même processus d'aller chercher une mémoire longue en Amérique et pas en Europe.

PO: Tout à fait. Et peut-être aussi de découvrir une autre temporalité, exactement comme on découvrait un autre espace. Le territoire de l'Amérique est tellement différent de celui de l'Europe par son caractère presque infini, sans borne. Le Brésil, comme le Canada, est un pays dont la superficie est impressionnante et qui a nécessité beaucoup de mobilité. Le peuplement d'un pays comme le Canada ou comme le Brésil nécessite des déplacements permanents. C'est pour ça que l'on a eu, nous, ce qu'on appelle les coureurs de bois qui faisaient de l'exploration, qui faisaient la traite avec les Indiens et qui ne tenaient pas en place. Nous n'avons pas eu une colonisation traditionnelle où l'on s'installait sur une terre qu'on cultivait pendant des années, des décennies, et qu'on se léguait de générations en générations. Les gens partaient, même les cultivateurs devaient partir l'hiver dans les forêts pour travailler comme bûcherons pour transporter le bois. Donc, ils quittaient leur terre. Il y a toujours eu cette idée du voyage, du déplacement, et on a cherché aussi une autre temporalité qui n'était pas celle de l'histoire européenne, qu'on peut faire commencer grosso modo à la Renaissance, qui a couru pendant l'âge classique et je dirais jusqu'à la décolonisation, jusqu'à ce qu'on a appelé le post-colonialisme, où là, on a commencé a remettre un peu en question aussi cette notion d'histoire, cette espèce de continuité progressant toujours vers l'idée absolue, vers la perfection. Une histoire au sens de Hegel, une sorte de progrès indéfini. Alors, les écrivains d'ici comme du Québec, je crois, ont cherché un autre temps. Un temps mythique plus qu'un temps historique.

ZB: C'est le hors-temps.

PO: Un hors-temps. Voilà.

ZB: C'est le titre d'un de vos livres théoriques.

PO: Oui, dans lequel j'expose un peu ce qu'on appelle aujourd'hui la posthistoire. J'essaie d'expliquer d'où vient ce concept et, sans doute, les chercheurs comme les écrivains ou les artistes qui ont promu ce terme là pour parler de notre temps ont eu conscience que l'histoire avait fait son temps, d'une certaine manière, qu'aujourd'hui on contestait beaucoup cette idée d'un progrès indéfini, ne serait-ce que pour des raisons écologiques où l'on voit que la technologie et la science ne peuvent pas progresser indéfiniment sans le respect de l'environnement. Ou pour des raisons économiques, on voit que la croissance des pays n'est pas indéfinie non plus. Cette idée d'une histoire sans cesse progressant est quelque chose qui est un peu en train d'agoniser, en train de mourir, au profit d'une autre temporalité qu'on recherche précisément dans le regard qu'on jette sur les sociétés préhistoriques. Il y a une analogie à faire entre cette entrée dans la posthistoire et ce regard qu'on peut avoir sur les sociétés d'avant l'histoire. Il y a d'ailleurs un anthropologue très important en France, qui s'appelle Pierre Clastres, qui est mort prématurément d'un accident, mais qui a écrit un grand livre qui s'appelle La société contre l'Etat et qui porte précisément sur la société tupiguarani qui est au Paraguay et en partie au Brésil, et qui montre comment ces sociétés ont créé des mécanismes pour empêcher l'instauration d'une histoire, l'instauration d'un état autoritaire, et, parmi ces mécanismes il y a ce qu'on appelle le chamanisme. Chez les tupi-guarani, les chamans sont appelés karai, ce sont des prophètes, artistes, écrivains, médecins aussi parce qu'ils connaissent les plantes médicinales, et dont la parole contrebalance toujours celle des chefs. Soit des chefs politiques, soit des chefs de guerre. Alors, il y a toujours un contrepoids, un contre-pouvoir qui est exercé par la parole, je dirais poétique ou artistique, incarné par le karaí. Pierre Clastres montre que, malheureusement, dans nos sociétés, il n'y a plus ce contrepoids, que l'imaginaire, qui a été d'une certaine manière abandonné par le discours religieux 
avec la sécularisation, la laïcisation, donc, l'imaginaire est resté orphelin, est resté sans aucune source d'inspiration, comme celles qu'avaient constituées les mythologies judéo-chrétiennes. Enfin, toute l'époque où le clergé avait une lourde influence sur l'imaginaire collectif. Donc, l'imaginaire est orphelin, du coup c'est l'art et la littérature qui auraient dû prendre la place, d'une certaine manière, des religions un peu défuntes, ce qui n'est pas arrivé. Je crois que depuis les années 50, 60, en France comme au Québec, malheureusement, la littérature et l'art on été un peu mis de côté au profit de ce qu'on appelle la société du spectacle, la société médiatique, dont le loisir, l'intertainment, le plaisir gratuit, purement ludique. Ce que Pierre Clastres disait, c'est qu'au fond, on devrait prendre modèle sur les sociétés tupi-guarani, par exemple, pour instituer une sorte de contre-pouvoir que les écrivains et les artistes pourraient exercer. Au Québec, on l'a fait un peu avec une génération d'artistes et d'auteurs qu'on a identifiée à un manifeste qui s'appelle le "Refus Global". Refus global, refus de tout ce que la société de consommation apportait déjà dans les années 50, l'américanisation progressive du Québec et du Canada. Ces artistes ont été à l'origine de ce qu'on a appelé la "révolution tranquille", un peu un paradoxe de parler de tranquillité...

ZB: Un oxymore.

PO: Oui un oxymore. Un paradoxe de parler de tranquillité à propos de révolution, mais c'est vrai qu'il n'y a pas eu vraiment de violence, sinon symbolique. Il y a eu une violence symbolique contre la domination du clergé, contre le pouvoir des classes dominantes qui étaient surtout anglo-saxonnes, britanniques et américaines. Donc, il y a eu une prise en main du destin du peuple canadien français, on ne l'appelait pas encore québécois, c'était le Canada français à cette époque.

ZB: C'est à ce moment là qu'on commence à parler du Québec, de littérature québécoise.

PO: Exactement. Même si le mot Canada a été inventé par Jacques Cartier, donc puisé dans les langues innues. Canada ça veut dire maison, habitation.

ZB: Aldeia.

PO: C'est ça, village. Et donc, c'était un mot que les canadiens français avaient inventé mais qui était un peu, je dirais, accaparé, pour ne pas dire volé, détourné.

ZB: Comme le mot Amérique, accaparé par les Etats-Unis. Nous tous sommes américains, eux ils sont étasuniens.

PO: Comme le mot Amérique, exactement. C'est ce qu'on devrait dire. Les artistes et écrivains, disons, ont tout de même eu un rôle majeur dans la transformation des mentalités et des sensibilités au Québec dans ces années 1950-1960 jusqu'au milieu des années 1970. Après, il y a eu un certain recul. Il y a eu de grandes figures comme Gaston Miron, grand poète, Hubert Aquin, grand romancier, Jacques Ferron, grand essayiste aussi, ou Pierre Vadeboncoeur, philosophe très important, Fernand Dumont aussi, qui ont contribué à cette révolution et, dès le début, beaucoup d'écrivains qu'on dit aujourd'hui migrants, qui sont arrivés au Québec dans les années 50-60, et qui ont aussi pris un peu en main le destin du Québec à partir de leur propres préoccupations. C'est, par exemple, le cas d'un écrivain iraquien juif, juif d'Irak c'est assez rare, qui est Naïm Kattan. Ces auteurs ont un rôle déterminant dans cette émancipation, je dirais, de la culture québécoise à partir d'un enrichissement de l'imaginaire qui s'ouvrait sur d'autres pays, le Moyen-Orient, l'Europe bien sûr, dont on avait quand même héritée et dont on avait un peu perdu l'héritage à cause de la conquête pendant deux siècles, on était un peu coupé de la France. Alors là, le fait qu'il y ait de plus en plus d'immigrants qui venaient du monde européen francophone a aussi contribué à cette évolution. Et puis, toujours l'intérêt, bien sûr, pour les sociétés amérindiennes, les premières nations. Ce mélange là a créé des conditions extrêmement favorables pour l'évolution des sensibilités. Mais je dirais qu'il y a eu une sorte de plateau qu'on a atteint au milieu des années 1970 et que, depuis, le redémarrage est plus difficile. On va en reparler plus tard. Donc, il y a eu cette espèce de contrepoids ou de contre-pouvoir que l'imaginaire littéraire a créé dans les mentalités et qui a, je pense, au Brésil comme au Québec, créé vraiment, je dirais un état de choc qui a permis une plus grande autonomie de nos sociétés.

ZB: En parallèle avec le modernisme brésilien.

PO: Voilà, exactement.

ZB: J'aurais une question qui a trait avec ce que vous avez dit sur les écrivains migrants. Je pense que c'est quelque chose qui intéresse énormément au Brésil et surtout à l'heure actuelle. Il y a beaucoup de recherches qui reprennent cette idée. Et votre livre, L'esprit migrateur, est très cité au Brésil, très utilisé par nos thésards et nos collègues chercheurs. Dans ce livre, vous allez au-delà du concept de littérature migrante, n'est-ce-pas? C'est un peu un contre, vous venez de parler de contrepoids, et vous allez au-delà du concept de littérature migrante pour mentionner que, par l'imagination, tous les écrivains sont migrants dans la mesure où ils peuvent aller chercher leur ancestralité culturelle dans les différentes cultures de la planète.

PO: Oui, d'abord, je me suis intéressé à cette notion de migrance parce qu'on lui a enlevé ses préfixes émigrants ou immigrants, où il était toujours question d'écrivains qui arrivaient, par exemple, sur le lieu donné, ou d'auteurs qui partaient vers d'autres lieus. Moi, je pense, qu'effectivement, il y a un aller-retour constant de tout écrivain, qu'il soit émigrant ou immigrant, entre 
l'ici et l'ailleurs. Nécessairement, quand on construit une œuvre, on déploie tout un monde imaginaire, on est attiré vers quelque chose qui est ailleurs, qui n'est plus ici. Je ne crois pas que la littérature se contente de reproduire l'ici maintenant. On est toujours en train d'inventer un autre espace, un autre... on pourrait dire un autre territoire, une autre histoire. Donc, on est, d'une certaine manière, un peu en errance, en immigrance, voilà. Notre écrivain national aujourd'hui, après la mort de Gaston Miron c'est Victor-Lévy Beaulieu. Lui, il parle de voyagerie. On parle d'imagerie par exemple ou de rêverie, ou de fantasmagorie, lui dit: "il y a les voyages, oui, bien sûr ça m'intéresse les voyages", bien qu'il ne soit pas, lui-même, un très très grand voyageur, il vit sur sa ferme. Mais il y a surtout les voyageries, c'est-à-dire, tous ces voyages qu'on entreprend par l'esprit, par la mémoire dans le passé, par l'imagination dans des temps alternatifs qui peuvent être l'avenir ou des présents autres que celui que nous vivons. Donc on invente.

ZB: Et de toutes façons, même chez les écrivains proprement migrants qui viennent des immigrations, l'imaginaire de l'ailleurs est aussi inventé.

PO: Tout à fait. Je crois qu'on parle beaucoup ici de Dany Laferrière qui a été traduit en portugais, et Dany explique très bien que le Haïti de son enfance, le pays de son enfance, il est réinventé.

ZB: il a passé trente ans sans y aller.

PO: Donc, il a développé une mythologie de son enfance. Joël Des Rosiers qui est un autre écrivain très important du Québec, né dans les Cailles, ces petits îlots qui sont au large d'Haïti et qui a une pensée un peu à la Edouard Glissant, c'est-à-dire une pensée insulaire. Il dit toujours que se sont des mondes qu'on ne peut pas réduire à leur géographie. Il y a toute une mythologie de la vie insulaire, de la vie sur l'île qui est irréductible, je dirais, à l'histoire factuelle de ces îles. Les gens inventent des contes, des légendes, ils ont des mythes de fondation qui sont complètement fabulés, qui ne reposent pas sur des faits historiques. Dans la propre autobiographie poétique de Joël Des Rosiers, tous ses poèmes sont autobiographiques, mais nourris par les mythologies qui viennent d'Afrique, qui viennent même du Moyen Orient. Dany Laferrière, même s'il utilise dans ses récits beaucoup d'anecdotes, donne une portée mythique à cette dimension. Régine Robin, qui est un autre écrivain qu'on a associé beaucoup à la migrance, invente son passé, elle se donne des pseudonymes. On sait qu'elle avait des parents polonais qui ont émigré en France, mais au delà des quelques faits qui sont à peu près avérés, il y a beaucoup de choses qui sont fabulées. Et, elle a un site internet où elle joue beaucoup de ses multiples identités. Alors, c'est pourquoi maintenant, on ne parle plus tellement d'auteurs migrants, on parle de littérature migrante, c'est la littérature elle-même qui est un processus de migration imaginaire, par l'esprit.

ZB: De n'importe quelle géographie.

PO: Exactement. C'est pourquoi on dit au Québec que toute forme d'expérience artistique, esthétique, littéraire poétique est, d'une certaine manière, un contact avec l'autre ou avec l'ailleurs. C'est un processus qui nous mène à une forme d'altérité, donc, ça participe des grands phénomènes qu'on connaît aujourd'hui de mobilité, de migrance. Le fait même d'écrire, toujours avec cette idée de la voyagerie, au sens de Victor-Lévy Beaulieu, c'est de partir pour ailleurs, s'établir sur un continent qui n'existe pas. C'est le continent de la langue, le continent de l'imagination, le continent de la mémoire, peu importe qu'on regarde vers le passé ou qu'on regarde vers l'avenir, on est toujours en partance. C'est une expression qu'utilise l'un des premiers poètes au Québec, Jean Aubert Loranger, dont je parlais tout à l'heure, et qui a inventé le terme, il n'a pas inventé le terme mais il a inventé la métaphore du passeur pour décrire l'écrivain. L'écrivain est un passeur qui va d'une rive à l'autre. Il y a toujours cette idée, vraiment, d'un esprit migrateur qui sous-tend la pratique littéraire ou la pratique artistique, qui est lié à l'invention de nouveaux territoires, pour l'imaginaire, ou à de nouvelles histoires, pour la mémoire à venir, et qui, toutes deux, histoire et mémoire, ou territoires et espaces sont faits de fantasmes, de mythologie autant que de faits avérés par l'historiographie.

Ana Maria Lisboa de Mello: Vous avez parlé de la communauté de mémoire, vous avez dit que ce n'est pas la même chose que la mémoire collective, vous pouvez en parler?

PO: Alors là, c'est pareil, parce qu'on a vécu beaucoup au Québec dans l'idée qu'il fallait développer ce qu'on appelle la mémoire nationale. C'était dans les années 1970, au moment où il $\mathrm{y}$ a eu un regain de nationalisme et un intérêt pour l'indépendance politique. Donc, on a beaucoup folklorisé, si vous voulez, la mémoire canadienne française, québécoise, en parlant effectivement, comment dire, des archives qui constitueraient notre passé et qui seraient exclusivement faites de ce qu'on a hérité de nos ancêtres français et puis de toute l'histoire du clergé, notamment, qui a dominé la culture québécoise pendant toute cette époque. Si bien que, par exemple, au musée de la civilisation à Québec, il y a une collection vraiment impressionnante de statues religieuses, de monuments, d'objets sacrés. On a axé la mémoire nationale sur ce passé-là et sur le passé rural aussi, alors, il y a beaucoup d'instruments agraires, d'instruments agricoles.

ZB: Maria Chapdelaine.

PO: Maria Chapdelaine, la littérature de terroir était très importante pendant toute cette période, même si 
dans la littérature régionaliste il y avait aussi des choses extrêmement intéressantes, y compris Louis Hémon. Mais il y a eu un excès, si vous voulez, de mise en valeur de ce patrimoine qui était lié vraiment à ce qu'on peut appeler l'enracinement dans la terre, l'identitaire pur. Comme ça, on aurait une seule lignée.

ZB: L'idée de racine unique.

PO: C'est ça, le sol et le sein comme on dit. Donc, une lignée assez linéaire justement, qui remontait à la nouvelle France.

ZB: Et le choix de Maria Chapdelaine de rester enracinée.

PO: Exactement, contrairement à François Paradis qui lui, comme les forestiers dont je parlais tout à l'heure partaient dans la forêt ou descendaient dans les grandes villes des Etats-Unis, etc., donc, lui était migrant. Je pense que la première vague de nationalisme au Québec était une vague plutôt d'enracinement dans la terre, dans les valeurs rurales et en réaction à cette vision purement nationaliste de la mémoire. Il y a eu cette idée, qu'au fond, il y a une communauté de mémoire. Personnellement, j'ai des ancêtres irlandais, par exemple, donc, au départ, anglophones qui se sont peu à peu francisés parce qu'ils étaient catholiques et qu'ils étaient d'avantage près, à ce moment-là, des canadiens français que des colonisateurs britanniques qui, eux, étaient tous pour la plupart protestants. Donc, par la religion, effectivement, les irlandais ont eu une influence très importante sur la culture québécoise. C'était, disons, déjà une mémoire musicale. On pense toujours que la musique qu'on dit canadienne, québécoise est purement autochtone. Non, c'est un mélange de musique celtique, de musique amérindienne. Donc, il y a eu comme une sorte de première hybridation, si vous voulez, déjà au début de la colonie. Et, on s'est dit peu à peu, qu'au fond, le Québec, le Canada français, ne pouvait s'établir que sur une communauté de mémoire, y compris les mémoires minoritaires, comme la mémoire juive. Vous savez que jusqu'à très récemment à Montréal, le Yiddish se parlait dans des quartiers comme Outremont. Les Hassidim sont très présents. Il y a eu un quotidien écrit en yiddish jusque dans les années 80 . Il y a eu des maisons d'édition en yiddish, etc. De grands écrivains de la communauté juive ont façonné l'imaginaire montréalais. De nombreux chercheurs, aujourd'hui, travaillent sur cet héritage. Il y a un gros travail sur la mémoire juive aussi à Montréal. C'est la plus grosse communauté juive "orthodoxe", comme on dit, avec beaucoup de guillemets, après celle de New York, de Brooklyn. En fait, après Brooklyn c'est Montréal qui a accueilli le plus de réfugiés venant des pays surtout de l'Europe de l'Est et puis de Russie. A partir des années 60, beaucoup du Maghreb aussi. La plus part des juifs pied-noir sont allés en France, mais un grand nombre est venu s'établir à Montréal. Donc la mémoire juive. De plus en plus ce qu'on appelle les mémoires amérindiennes, les premières nations qu'on découvre. Moi, j'ai été pendant quelques années professeur à l'Université de Chicoutimi qui est dans le Moyen Nord. C'est presque la taïga. On était environné de réserves amérindiennes dont celle des Innus de Mashteuiatsh avec lesquels on a eu des contacts très très étroits. Nous avons été la première université, la première faculté, a donner des cours d'innu, des cours de grammaire et des cours aussi sur la littérature orale innue, qui a été recueillie d'abord par les missionnaires, ensuite par les ethnologies. Et, à notre grande surprise, dans cette université, on accueillait des gens de partout au Québec qui venaient étudier cette culture, il y avait beaucoup d'innus également, qui avaient oublié leur propre langue, oublié leurs traditions, qui avaient été acculturés complètement par la culture dominante.

ZB: Inuit c'est une chose, Innu une autre.

PO: Les Inuits sont plus au Nord. Les Innus se sont les Montagnais, ce sont des Indiens, pas des Esquimaux. Il n'y a pas beaucoup de relations entre eux. Il y a un très vaste territoire qui les sépare. Mais c'est vrai qu'il y a presque une homophonie entre Inuit, Esquimaux on refuse maintenant d'utiliser le mot esquimaux qui voulait dire mangeurs de chair crue et c'était presque une insulte -, et Montagnais, ceux qui vivaient dans les montagnes. Montagnais est un mot français, ils s'appellent Innus et en Innuktitut ça veut dire hommes, on est des hommes. Innuktitut c'est la langue des innus. Il y a une grande poète qui s'appelle Joséphine Bacon, c'est un nom qu'on lui a donné mais ce n'est pas son vrai nom. Je ne pourrais pas vous donner son nom en innu car c'est très long. C'est un nom que les anglophones lui on donné: Bacon, très grand poète innue qui a publié plusieurs livres. Elle est finaliste du grand prix de la ville de Montréal pour son dernier livre. Donc, tout ça pour vous dire qu'il y a eu un progrès énorme, ce département d'anthropologie a été créé il y a vingt-cinq ans à peu près, et on a accueilli beaucoup d'innus qui sont venus se former à leur propre culture à travers les travaux des anthropologues.

ZB: Et ils constituent des communautés de mémoire.

PO: Exactement. Ces cultures, les nouveaux arrivants, comme les Juifs d'Europe de l'est, les cultures des premiers migrants comme les irlandais qui sont arrivés, ensuite les italiens, les portugais, il y a un quartier portugais important sur le plateau de Montréal; tout cela a constitué une mémoire multiple qu'on appelle maintenant une communauté de mémoire. Donc ce n'est pas une mémoire commune parce qu'elle appartient à plusieurs traditions, plusieurs histoires différentes, plusieurs lieus où elle s'est développée, mais le fait que tous ces gens, maintenant, participent de la société québécoise, ou canadienne française, fait en sorte que l'on 
vit dans la communauté de mémoires. On adopte, d'une certaine manière, l'héritage des juifs d'Europe de l'Est. Maintenant, aussi beaucoup de personnes de l'Asie du Sud Est. Par exemple, tous ces vietnamiens qui sont partis dans les années 70, que l'on a appelés les boat-people, et qui ont été accueillis massivement au Québec, ont constitué aussi une littérature importante. Il y a Kim Thuy, qui est très célèbre au Québec et qui a écrit des livres sur son expérience de la migration très difficile à travers cette épreuve des boat-people. Donc, on a adopté toutes ces mémoires, c'est pourquoi vous avez certainement entendu parler de la fameuse Charte des Valeurs du parti québécois qui fait la promotion de l'indépendance. Cette Charte des Valeurs a soulevé un tôlé de protestations parce qu'elle était trop centrée, encore une fois, sur l'idée qu'on avait qu'une seule histoire.

ZB: Et de la laïcité.

PO: Et de la laïcité, c'est-à-dire que les gens ne veulent pas que l'on perde la mémoire des grands textes sacrés qui ont été fondateurs, non seulement, de notre peuple mais de tous ceux qui sont venus l'enrichir. Si vous vous promenez dans Montréal par exemple, vous voyez à la fois des mosquées, des synagogues, des églises protestantes de différentes confessions, anglicanes, évangélistes, et puis des églises catholiques bien sûr. Et on veut préserver cet héritage là, et on veut que sur la place publique continue, d'une certaine manière, d'y avoir des signes qui témoignent de cette mémoire là. Donc, on ne veut pas éradiquer toutes les histoires que les émigrants ont apportées pour constituer le tissu complexe du Québec contemporain. Alors, heureusement, cette Charte des Valeurs a été mise un peu de côté, même Gérard Bouchard, qui est considéré comme un nationaliste, a pris fait et cause contre cette charte disant qu'elle était dangereuse dans la mesure où elle voulait resserrer ce qui était des valeurs communes. Ce qu'on appelle, je dirais, la culture collective au Québec est quelque chose qui ne s'enferme pas dans des murs ou dans des frontières, qui ne peut pas être considéré comme nationaliste, l'apport des autres cultures fait partie du bien commun. On veut conserver cette idée là d'une ouverture. C'est un peuple qui a vécu essentiellement d'immigration depuis une trentaine d'années. Le taux de natalité est très faible, donc, s'il n'y a pas cet apport, d'une certaine manière, il y a une progressive disparition du Québec. Je pense que ça a été un débat extrêmement intéressant.

AMLM: Et on partage la mémoire des autres, c'est pour ça que vous avez dit: "Je me souviens de ce que je n'ai pas vécu".

PO: C'est vrai, exactement, parce qu'évidemment, comme on était en Amérique, on était loin de la dernière guerre. Moi, je suis né, je raconte ça dans un roman qui est paru, qui s'appelle Portrait de dos, je raconte un peu cette première enfance. Je suis né au milieu du siècle, donc en 1950, cinq ans seulement après la fin de la Seconde Guerre mondiale dont on ne parlait pas du tout en Amérique. Pendant toute mon enfance, on a fait comme une espèce de silence sur ce qui c'était passé. Par contre, on parlait beaucoup de la guerre froide, peutêtre ici aussi, mais pour nous, à cause de la proximité du Canada avec les Etats-Unis, il y a vraiment eu un traumatisme. On connaissait la bombe atomique, on connaissait tout le conflit lié à l'installation des missiles russes à Cuba. Donc, il y a eu une très grande inquiétude dès 1955 jusqu'en 1965 à peu près, ça a duré dix ans, grande inquiétude face à une éventuelle guerre nucléaire qui toucherait le Canada, peut-être en premier, avant les Etats-Unis. Les gens construisaient des abris, on faisait des exercices etc. Il y avait une sorte de traumatisme chez tous les jeunes enfants qui sont nés dans ces années là, ce qui a éveillé le souvenir de la dernière guerre. On parlait beaucoup de Hiroshima et Nagasaki bien sûr, mais très peu de Auschwitz, Treblinka, de l'extermination des juifs d'Europe.

ZB: Et on parle encore beaucoup de la Première Guerre parce que le Canada y a eu une présence importante.

PO: Tout à fait. Alors, ces traumatismes liés à la guerre froide ont fait naître une sorte d'inquiétude face à toute forme d'extermination et, du coup, ma mémoire s'est élargie peu à peu par les lectures que j'ai faites, par les films que l'on voyait aussi. Donc, j'ai eu très très tôt une espèce de sentiment de culpabilité d'avoir participer indirectement par le silence de l'Amérique, du Canada en particulier sur l'extermination des juifs, d'avoir participé à cette horreur qu'à été la Shoa. Une culpabilité et en même temps une culpabilité face au silence dans lequel on tenait cet évènement si tragique. On trouve cela pas seulement chez les écrivains, il y a une conscience très vive des canadiens, c'est peut-être la raison pour laquelle, pendant très longtemps, le Canada a été considéré le pays exemplaire pour ce qu'on appelle les missions de paix. Vous savez que le Canda est à l'origine des fameux Casques bleus de l'ONU. C'est Lester Bowles Pearson, premier ministre du Canada à la fin des années 60 , qui a créé cette idée des peace corps et a d'ailleurs reçu le prix Nobel de la paix pour cette action. Cette conscience là est très vive, si bien qu'il y a des mémoriaux sur l'extermination des Juifs d'Europe un peu partout au Canada. Il y en a un qui va être construit très bientôt à Gatineau en face d'Otawa. Le fait qu'il y ait une communauté juive très importante au Canada a contribué aussi à cet intérêt pour marquer cette mémoire là. Mais je pense qu'au Québec en particulier, il y a une mémoire très vive de ça, qui est indirecte, d'une certaine manière, qui est passée par la parole, par les images et par la littérature, beaucoup. 
S'il n'y avait pas eu toute cette littérature... On pense notamment à Naïm Kattan qui a conscientisé les gens, Mordecai Richler en particulier ou Léonard Cohen, qui sont tous des montréalais.

ZB: Régine Robin.

PO: Régine Robin. Régine est arrivée un petit peu plus tard. Mais, même tardivement, elle y a beaucoup contribué. Ce qui fait que, d'une certaine manière, on s'est approprié cette mémoire, mais sans en prendre possession non plus, disons qu'on s'est laissé imprégner plutôt par la mémoire de l'autre. Ce qui fait que notre mémoire, maintenant, n'est pas purement identitaire, elle est beaucoup liée à cette idée qu'on est tous, d'une certaine manière, un peu autre. On n'est jamais totalement soi, on est rempli d'hétéronymes en nous qui parlent aussi à notre place.

ZB: C'est pour ça que vous dirigez la collection "Soi et l'autre".

PO: "Soi et l'autre", et même comme écrivain, je dois vous dire que ce n'est pas seulement comme théoricien, je me sens habité par plusieurs voix et, quand j'écris, je ne suis pas seul à parler, c'est une écriture chorale, je fais parler en moi toutes ces voix qui m'ont habité, grâce à ce que j'ai entendu, à ce que j'ai lu. Je fais parler aussi les voix de ceux ou celles à qui je m'adresse. Je fais parler les voix de ceux ou celles avec qui je vis, dans ma vie à la fois personnelle, familiale et aussi collective. Ce sont toujours des identités multiples qui s'expriment, à mon sens, dans l'écriture romanesque ou l'écriture poétique, et c'est la raison fondamentale pour laquelle je me suis intéressé à ces questions d'un point de vue plus théorique. C'est vraiment cette expérience, tout part non pas d'une idéologie ou d'une morale, de l'altérité qui devrait être le plus largement partagée, mais plutôt d'une expérience vécue ou du fait qu'on est autre nous-même. C'est l'expérience que Rimbaud a vécu en disant "je est un autre", et l'intérêt que j'ai manifesté en tant que théoricien, en tant que critique, pour cette littérature migrante, cette littérature de la mobilité vient d'un sentiment profond, j'allais dire d'un affect même, lié à cette idée qu'on est profondément autre. Intérieurement autant que dans nos contacts avec autrui.

Marie-Hélène Paret Passos: J'ai vu dans vos écrits, cette place, cette inquiétude et cette mémoire de la Shoa. Dans ce livre: Où suis-je. Parole des égarés, vous parlez aussi de l'importance de la voix. Je me demande dans quelle mesure est-ce-que ce lieu de présence ne serait pas un désir de retour à cette parole prophétique, la parole prophétique telle que Maïmonide l'évoque justement dans Le Guide des égarés?

PO: Tout à fait, vous avez bien raison parce qu'effectivement, il y a une dévalorisation aujourd'hui de la voix au profit de l'image, de l'image médiatique. C'est- à-dire que maintenant tout doit être visible alors que la voix est quelque chose de plus mystérieux. Même s'il y a un interdit de la représentation dans plusieurs religions monothéistes, on pense à l'islam et au judaïsme, la voix reste omniprésente. Il y a la voix de Dieu qui se manifeste auprès de Moises quand il s'agit de léguer la Loi, ce qu'on appelle le Décalogue, les Commandements, donc quelque chose comme ça qu'on ne voit pas, qui est une présence mystérieuse, qui est une voix qu'on entend. Moi j'aime beaucoup aussi l'idée du souffle. On emploie souvent ce terme dans les textes sacrés pour la voix, c'est-à-dire quelque chose qui est de l'ordre d'une respiration dans la langue, dans la parole, qui est unique mais universelle à la fois. Chacun a une voix, mais en même temps on parle de la Voix, avec un grand V, comme l'expression de l'oralité pure qui passe donc à travers le souffle, respire. Respire c'est spiritus, c'est le même mot qu'esprit et c'est le mot aussi qui va donner le mot inspiration. Quand je disais tout à l'heure qu'il y a plusieurs voix en moi qui parlent, et bien, ça renvoie un peu à cette idée traditionnelle qu'on avait de l'écriture poétique ou romanesque comme étant une écriture inspirée, respirée etc., et les prophètes disent toujours dans les textes sacrés, que ce soit la Tora ou le nouveau testament, ils disent toujours: "J'ai reçu la parole de Dieu", il y a quelque chose d'autre en moi qui a parlé et moi je ne suis qu'une voix de passage, le passeur qui finalement transmet la parole. Je suis un transmetteur, et je pense que tous les écrivains, d'une certaine manière, sont des transmetteurs, des espèces de courroies de transmission qui font venir en eux la voix des morts, de tous ceux qui sont disparus, donc la voix des anciens, etc., et qui les transmettent à ceux qui ne sont pas encore, à ceux qui viennent, à ceux qui vont prendre la parole un jour, et donc c'est toujours l'idée du passage de témoin. On passe un témoin, c'est aussi une autre caractéristique très forte de l'écrivain, c'est celui qui porte témoignage. Et le mot testament, le mot témoignage sont reliés, c'est la même étymologie latine. Donc, la parole littéraire est à la fois testimoniale, parce qu'elle garde quelque chose du passé, comme si elle avait une fonction conservatrice, d'une certaine manière, pour que les choses ne se perdent pas, mais elle est aussi testamentaire, c'est-à-dire qu'elle lègue pour l'avenir, donc elle est tournée vers ce qui vient, ce n'est pas juste de l'archivage, ce n'est pas juste de la muséologie, conserver pour conserver, ce qui serait momifier, fossiliser. C'est plutôt faire renaître, quand on lègue quelque chose c'est qu'on veut que le vivant puisse s'en servir, donc il faut que ce soit encore, réutilisable dans la vie des gens. Il ne faut pas que ce soit quelque chose de figé. C'est vraiment l'idée d'une renaissance, d'une résurrection du passé vers un avenir, qui est dans la parole littéraire comme un témoignage et testament. Quelque chose qu'on laisse passer mais qu'on rend vivant, 
même si en apparence c'est quelque chose qui apparaît mort, qui semble mort. Donc, tous ces grands mythes qui sont fondateurs de nos sociétés - je pense surtout aux grands mythes monothéistes -, on a un rôle majeur, je pense, en tant qu'écrivain, qui est justement de les faire renaître autrement.

ZB: Transmission.

PO: La transmission, ça ne veut pas dire les transmettre à la lettre, pour qu'on les respecte de manière dogmatique, mais pour en transmettre l'esprit. C'est pour ça, encore une fois, que je parle d'esprit migrateur, pour qu'aujourd'hui on voie comment on peut refonder autrement ces mythes là. Récemment, Anne Elaine Cliche qui est professeure dans mon département, et écrivain, a écrit un très beau livre qui s'appelle Jonas de mémoire, elle a écrit un autre livre autour de Job. Elle reprend tout le mythe de Jonas et elle l'applique au monde contemporain. Elle en fait un vrai personnage de récit moderne, mais avec toutes les connaissances qu'elle a de la Tora et avec toutes les interprétations, après, qu'on en a données avec le christianisme. Alors, il y a une profondeur historique, presque légendaire dans sa parole, mais en même temps c'est un Jonas qui travaille à l'usine, donc, il y a une transposition, une revitalisation, une revivification $\mathrm{du}$ mythe ancien. Ce n'est pas pour qu'on les respecte dans leur lettre mais pour qu'on comprenne leur esprit. Il y a une migration de l'esprit biblique dans la littérature contemporaine. Il y a un personnage de Victor Lévy Beaulieu, que je cite encore, et qui s'appelle Job J Jobin.

ZB: Moacyr Scliar faisait ça aussi.

PO: Alors je ne sais pas si ça répond à votre question, mais c'est par la voix, d'une certaine manière, porteuse de cet esprit, qu'on peut faire entendre ces choses là, l'importance des grands mythes fondateurs.

MHPP: Et, qui dit voix dit écoute.

PO: Oui, exactement.

MHPP: Je voudrais ici introduire le nom d'Henri Meschonnic pour qui l'important était de traduire cette parole dans l'écrit, ce qu'il appelait le rythme.

PO: Tout à fait.

MHPP: Comment replacer le mouvement de la parole dans l'écriture, je pense que nous sommes au cœur du sujet. Henri Meschonnic parlait aussi de distance entre le mythe ancien et sa réactualisation, ce qui est bien illustré dans ce que vous avez cité. Jonas qui est réactualisé, ce qui prend en compte toute cette distance et qui fait partie de la transmission.

PO: Voilà. Vous savez, j'ai participé, j'ai eu le plaisir de participer à la traduction de la Bible des écrivains de Bayard. Je ne sais pas si vous la connaissez, qui a suscité une polémique avec Henri Meschonnic, que j'ai bien connu, et avec d'autres aussi. J'ai donc traduit deux petits prophètes. Esdras et Tséfania qu'on traduit par Sophonie dans la tradition latine, et j'ai traduit aussi de larges parties de Jérémie. Malheureusement, je n'ai pas eu le temps, à cause de mes fonctions à l'université, de terminer. C'est François Bon, l'écrivain français bien connu qui a fait la suite. Mais on m'a demandé de poursuivre ma traduction et on va la publier séparément.

ZB: Vous avez traduit à partir de quel livre?

PO: Alors, je vais vous expliquer comment cela c'est passé. On était couplé, ou jumelé, si vous voulez, avec un bibliste, donc un philologue, qui connaissait soit l'araméen, soit l'hébreu, soit le grec, indépendamment de la langue d'origine, et qui nous fournissait à la fois une sorte de traduction littérale et puis surtout une translittération de l'alphabet hébreu. Dans mon cas, c'était deux textes hébreux, en alphabet romain. Donc, j'ai pu lire la phonétique du texte hébreu original.

ZB: Quel travail!

PO: Ça a été un travail énorme parce que chaque fois, on devait justifier chaque verset qu'on traduisait devant une assemblée de biblistes qui venaient de France et du Québec. J'avais l'impression d'avoir fait quatre soutenances de thèse, simplement pour ces deux petits textes, car c'était vraiment un affrontement constant. Mais je dois vous dire une chose, c'est qu'avec le bibliste qui m'a accompagné dans la traduction de Tséfania, Sophonie, j'ai eu un énorme plaisir. D'abord, parce que c'est quelqu'un que j'ai pu fréquenter sur une base quasi quotidienne, il vivait à Montréal. Grand spécialiste non seulement de la Bible mais de l'hébreu, il m'a donné beaucoup de liberté pour traduire mon texte au plus près de la translittération en alphabet romain du texte hébreu, donc de l'oralité. Il était très sensible à l'oralité. Je ne sais pas si vous savez que dans ce texte là, de Sophonie, on trouve le fameux dies irae, jour de colère, qui est un texte très violent, où Adonaï, Yahvé dit qu'il va détruire le peuple d'Israël s'il ne se repend pas de ses fautes. Donc, c'est un texte de colère, un peu comme Jérémie, ou Isaïe, et il faut que ce soit proféré, il faut que ce soit quelque chose qui s'entende.

ZB: Fort.

PO: Voilà. Malheureusement, je ne l'ai pas avec moi, j'aurais pu vous le lire parce que ça été même mis en musique par des rappeurs, ce petit texte que j'ai écrit autour du dies irae est vraiment très sonore. Donc, c'était un plaisir pour moi de travailler sur Tséfania, qui est un grand prophète même si le texte qu'on a gardé de lui dans la Tora est très court. Pour moi, il a la force de Jérémie, d'Ezéquiel, de Qohélet, etc. Par contre, pour Esdras qui est plus un chroniqueur, plus un historiographe qui a raconté l'histoire du roi David, ça a été beaucoup plus difficile parce que l'oralité était moins évidente. Et, j'ai travaillé avec un bibliste français qui était éduqué dans la tradition de ceux qui on traduit la Bible de Jérusalem, donc, une tradition vraiment chrétienne, où c'est l'écrit 
qui prime sur la profération. Alors là, j'ai été beaucoup censuré parce qu'il y avait nombre d'expressions chez Esdras où l'on disait par exemple, et c'est très littéral, je me suis beaucoup fondé sur la traduction littérale, mais en lui ajoutant quelque chose qui était la force poétique que j'essayais de retrouver dans le texte. Par exemple Esdras dit souvent: " $j$ 'ai pris de ma main les bouches dans la main d'Adonaï je les ai mis dans ma bouche et je vous les restitue" mais il dit littéralement: "je vous les crache". Alors j'ai utilisé des mots assez crus, proches de la langue populaire et ça a été censuré. On ne l'a pas accepté, mais ce sont des choses vraiment propres à l'oralité que j'ai cherchées, vraiment dans l'orientation d'Henri Meschonnic qui privilégie toujours les rythmes poétiques aux rythmes rhétoriques. Les rythmes rhétoriques sont ceux qu'on hérite de la tradition pure et qu'on reproduit. Les rythmes poétiques sont ceux qu'on réinvente constamment. Donc, j'ai essayé de réinventer de texte biblique, comme beaucoup d'autres on fait. Je vous recommande cette Bible, il y a une traduction merveilleuse de Frédéric Boyer de la Genèse, de Jacques Roubaud pour Qohélet justement, la traduction est merveilleuse. J'en cite un passage au tout début de Portrait de dos. Il y a une réinvention de la parole biblique de sorte que, même si cette Bible n'a pas reçu l'imprimatur du pape et de la congrégation vaticane, elle est beaucoup utilisée au Québec dans les offices religieux, tout le long de la côte de ce qu'on appelle la rive sud.

ZB: Je n'avais pas compris qu'elle aurait cette fonction, je pensais que c'était plutôt quelque chose de littéraire.

PO: C'est littéraire mais c'est une vraie Bible. Ça a été publié par Bayard en France et Médiapaul au Québec. Vous avez ici les éditions Paulinas qui sont reliées aux éditions Paulines, tous ont donc participé à ce projet de traduction. On l'a appelée la Bible des écrivains parce qu'il y a de grands écrivains comme Pierre Michon, Jean Echenoz, Jacques Roubaud, Emmanuel Carrère, des écrivains très importants. C'était très conservateur, c'està-dire que n'ont été gardés que les livres canoniques de ce qu'on appelle l'Ancien et le Nouveau Testament. On voulait, nous, ajouter tous les testaments apocryphes de saint Thomas, de Maria de Magdala, etc. On aurait voulu faire quelque chose de beaucoup plus important. Je crois que Frédéric Boyer a l'intention de créer un deuxième tome avec tous les évangiles apocryphes. Donc, on va poursuivre l'expérience. Mais, effectivement, je crois que le Vatican a trouvé qu'il y avait trop de liberté qui avait été prise face à la lettre, et surtout disons, face à la tradition chrétienne de traduction, nous avons été probablement trop hérétiques pour eux. Mais, cela dit, je peux vous dire que le clergé au Québec utilise abondement cette Bible, même si elle n'a pas reçu l'autorisation papale.
MHPP: C'est ce que Meschonnic appelle la censure Théologico-politique.

PO: Exactement.

ZB: J'aimerais parler un peu de votre immense capacité de production intellectuelle qui va de la poésie au roman. Il y a aussi énormément d'essais littéraires et philosophiques. Pendant cette période de votre présence à la Foire du Livre, on a eu l'occasion d'en parler. Je connaissais votre très importante production intellectuelle, mais je ne savais pas que ce sont 52 livres signés par vous. Donc, c'est vraiment une chose différente, disons, de la majorité des auteurs de votre génération, cette production tellement importante. Et, vous avez dit que l'écriture devient pour vous vraiment un impératif. C'est une sorte de guérison, une psychanalyse, vous pensez pendant la nuit et, le matin, ça sort. J'ai très envie de savoir.

PO: Oui, je vous le disais, mon dernier livre s'appelle De l'air. Pour moi, l'air n'existe pas sans la parole, sans le souffle. Pour moi, l'élément dans lequel je vis c'est l'air en tant qu'il est habité par les mots, par les voix, par les paroles. On pourrait même dire aussi par les chants d'oiseaux, par le bruit, les feuilles sous le vent, par tout ce qui bruisse dans la nature et dont la voix fait partie. Pour moi, prendre la parole ce n'est pas quelque chose qui est à part du fait, par exemple, je ne sais pas, que les lions rugissent, que les chiens aboient, c'est quelque chose qui est propre aux êtres vivants de proférer quels que sons que ce soient. On profère des sons. Et pour moi, la littérature c'est le son par excellence, exactement comme on a l'impression que le chant pour les oiseaux est quelque chose de vital. Un oiseau qui ne chante pas est un oiseau qui va mourir. Il faut que quelque chose soit proféré, d'une certaine manière, et je le fais bien sûr par écrit. Mais j'ai une longue habitude, parce que vous voyez, le Québec c'est un peu comme le Brésil, on voyage beaucoup, j'ai donné beaucoup de cours à Chicoutimi tout en vivant à Québec et en ayant une compagne qui habitait Trois Rivières. Donc, j'étais beaucoup sur la route et perdait beaucoup de temps en traversant la forêt, parce qu'on traverse le parc des Laurendites, c'est très long, il n'y a rien, que des arbres et des orignaux. J'écrivais mentalement pendant ces voyages et il fallait que ces phrases qui me traversaient l'esprit soient bien calibrées sur le plan musical, sonore pour se graver dans ma mémoire. Il fallait vraiment que ce soit très euphonique pour qu'elles puissent, comment dire, perdurer pendant tout le voyage, qui dure presque trois heures. Souvent j'arrivais très tard, et il n'était pas question pour moi de prendre la plume ou de me mettre à l'ordinateur pour les transcrire, donc j'attendais le lendemain matin. Alors, ça passait par le rêve, etc. Et, dès le lendemain, je restituais toutes ces pages, en général deux, que j'avais écrites pendant le voyage et qui avaient un peu été 
métamorphosées par le travail onirique de la nuit, mais c'était quelque chose de déjà extrêmement structuré. Pendant ces longs itinéraires, je scandais mes mots, je les reprenais, ce sont des procédés mnémotechniques en fait, le travail de versification. J'ai fait ça à la fois pour la prose, pour l'essai et pour la poésie, c'est ce qui fait que le rythme est très important, y compris dans mes essais, ce travail de la langue, de la prosodie. Pour moi c'est essentiel à la transmission de ce que j'ai à dire. Ce que j'ai à dire, ce ne sont pas uniquement des idées, des concepts, des notions, bien sûr c'est présent, mais c'est aussi quelque chose d'autre qui passe d'avantage par la sensibilisé par l'émotion, par l'affect et, à mon sens, la vocalité, la prosodie, la rythmique des phrases, des vers, transmet ce genre de chose. Une dimension plus affective de la pensée qui n'est pas réductible aux notions de concepts que j'ai mis en place. Il faut dire que j'appartiens aussi à une génération qui a été formée à l'écoute de Roland Barthes, de Jacques Derrida, de Michel Foucault, qui étaient tous aussi de grands écrivains. La pensée n'était pas réductible à une approche strictement scientifique ou technique, elle était portée aussi par quelque chose qui était une sorte de souffle. J'ai eu la chance d'entendre Lacan dans ses séminaires et je peux vous dire que c'est quelqu'un qui avait une parole, même si parfois on ne comprend pas toujours très bien, même si on se demande quel sens il véhicule, il avait un pouvoir de fascination par sa parole. L'énigme de la voix c'est quelque chose qui pour moi est très important et qui est le propre de ce qu'on appelle le style chez un auteur. Cette petite musique dont parlait Louis Ferdinand Céline, c'est quelque chose d'un peu énigmatique, mystérieux, quelque chose qu'on ne comprend pas tout à fait mais qui fait passer tout le reste, dont on a besoin, absolument.

ZB: Vous êtes en train d'écrire une trilogie, Portait de dos, c'est le premier?

PO: C'est le premier, le deuxième va paraître au printemps, et s'appelle Dans le temps. Alors, dans le temps c'est une expression populaire: dans le temps on a vécu telle chose, et ça veut dire aussi littéralement: dans le temps, on habite le temps. Portrait de dos traitait de l'enfance tardive et de la jeune adolescence, le deuxième tome traite plutôt de la jeunesse.

ZB: Portrait de dos ça veut dire que vous parlez de choses qui sont une découverte?

PO: De choses qui sont dans mon dos, que je ne vois pas. Je dis toujours que c'est une autobiographie non autorisée, comme si on avait écrit contre mon gré cette espèce d'autofiction.

AMLM: Ça m'intéresse beaucoup.

PO: Voilà, donc cette idée de l'autobiographie non autorisée c'est une sorte d'autofiction qui s'écrit contre son propre gré, qui ne présente pas un autre visage mais quelque chose qui est caché, qui est secret et qui est presque sacré dans certain cas, parce que le mot secret et sacré sont liés. Donc, il y a quelque chose dont on ne doit pas parler, il y a une sorte d'omerta qui touche ce passé là, donc, ça m'arrive dans le dos, un peu comme on dit qu'on poignarde quelqu'un dans le dos, qu'on attaque quelqu'un dans le dos. J'ai été attaqué dans le dos par une sorte de narrateur omniscient, qui sait tout de moi, alors que moi je fais comme si je ne savais rien. Le personnage qui s'appelle Jean Lhomme, qui est un peu mon alter ego, ne prend presque jamais la parole, c'est un narrateur anonyme qui parle pour lui et qui est précisément celui qui me trahit, me traduit en parlant dans mon dos. Ça se dit aussi peut-être en portugais, au Québec: il parle dans mon dos, ça veut dire qu'il me calomnie, qu'il commet des médisances etc.

ZB: Dans le temps, va paraitre en 2015?

PO: 2015, oui c'est ça, probablement au printemps. C'est un livre beaucoup plus volumineux parce que cette période de ma vie, entre l'âge de 18 et 32 ans à peu près, a été assez complexe, avec des ramifications dans le monde politique. Et je traverse beaucoup de ce qu'on appelle aujourd'hui les xénomythologies, c'est-à-dire comment le Québec de ces années là - c'est le moment qu'on appelait la contre culture, je ne sais pas si ça a été vécu ici -, découvrait les cultures autres. Moi, j'ai fait la découverte du taoïsme, du bouddhisme, à cette époque là, ça arrivait un peu par le bais de ce qu'on appelait la nouvelle gauche, qui était californienne, à travers l'université de Berkeley, des gens comme Marcuse, comme Castañeda, comme Mircea Eliade. Donc, on découvrait toutes sortes d'autres mythologies que celle dans laquelle on avait vécu par l'éducation qu'on avait reçue. Je raconte comment, finalement, le taoïsme, le bouddhisme ont pu influencer ma pensée à cette époque là. C'est un livre qui est à la fois un livre, je dirais, ingénu parce que j'essaie de me placer dans la position de celui qui a vécu ça au départ, au moment où on découvrait les choses, mais en même temps érudit, parce que maintenant que je connais bien tout le travail que ces mythologies ont fait en profondeur sur l'imaginaire occidental, je peux mieux comprendre la portée que ça a pu avoir. Donc, j'apporte des éléments dont je n'aurais pas pu être conscient à l'époque. Maintenait le Tao m'est très familier parce que je l'ai beaucoup utilisé dans ma création, je peux donc voir la portée que ça avait.

ZB: C'est un legs de ce vécu aux générations d'aujourd'hui.

PO: Voilà, exactement, et surtout pour montrer comment on vit les faits, c'est une première chose. Bien sûr, il y a des anecdotes qui sont liées à ce que j'ai vraiment expérimenté, éprouvé. Mais on vit aussi des fictions, dans toutes les époques de notre vie il y a une espèce de pas que l'imaginaire prend dans l'histoire 
personnelle, dont il faut tenir compte, dans toute forme d'autofiction ou d'autobiographie. Alors, c'est cette dimension fictionnelle, un peu dans mon histoire, que je veux léguer aussi, tous les mythes qui ont accompagné mon enfance et ma jeunesse.

MHPP: Pour revenir à votre processus d'écriture, d'après ce que vous disiez par rapport aux voyages, j'ai l'impression qu'en ce qui concerne votre processus de création, le processus mental a une grande part, puisque vous dites que lorsque ça sort c'est pratiquement structuré.

PO: Oui, exactement. C'est-à-dire que je ne suis pas du tout du genre un peu tâcheron, à me mettre devant la page blanche et attendre que les mots sortent. Il y a tout un travail préliminaire au moment de l'écriture que j'appelle moi de l'inscription, j'inscris ce qui est déjà écrit, souvent de manière purement mentale, dans mon esprit. Il y a tout un travail, je dirais plus spirituel, qui prépare, qui accompagne le geste d'écriture, qui devient presque un geste technique, a un moment donné ça devient une transcription.

MHPP: Vous écrivez à la main, à l'ordinateur?

PO: Quand je suis à la maison à l'ordinateur, quand je suis en voyage c'est plutôt à la main.

MHPP: Vous imprimez quand vous écrivez à l'ordinateur? Vous relisez sur papier?

PO: Oui, bien sûr oui, je me relis sur papier parce qu'à l'écran il y a quelque chose qui échappe, oui, j'ai besoin de voir l'imprimé.

MHPP: Mais pas tout le monde ne le fait, aujourd'hui.

ZB: Donc vous allez laisser du matériel pour les gens qui font de la génétique.

PO: Et bien, c'est-à-dire que je ne garde pas toutes les versions bien sûr.

MHPP: Vous ne gardez pas tout?

PO: Non, je ne garde pas toutes les versions, mais souvent les épreuves. Je travaille au moins sur trois jeux d'épreuves parce que je fais des corrections jusqu'à la dernière minute, et je garde mes jeux d'épreuves. C'està-dire, qu'une fois que c'est entre les mains de l'éditeur, en général, il m'envoie une première copie tapuscrite annotée de sa main et moi-même je l'annote. Je garde ces choses là pour des raisons plus autobiographiques, j'ai besoin de savoir comment mon texte a évolué dans le dialogue avec l'autre. Et puis après, les différentes épreuves imprimées, en général il y en a trois, sont aussi régulièrement annotées.

ZB: Trois, c'est très minutieux.

PO: Oui, contrairement à ce que les gens pensent, même si je publie beaucoup, je prends un soin énorme et je prends beaucoup de temps à faire un livre. Ce n'est jamais quelque chose de spontané. Dans le temps, je l'ai remis au mois d'août dernier et on continue à travailler sur le livre. Tant que je ne suis pas satisfait, on retarde la publication. Donc, parfois les livres paraissent deux, trois en même temps parce que j'ai mis trop de temps à remettre les épreuves. Je travaille beaucoup sur épreuves, je garde les épreuves, c'est ça qui à mon sens témoigne un peu du travail génétique si vous voulez, parce que les premiers manuscrits, je sais que ça peut être important pour quelqu'un qui fait des cherches, mais, en général, il y a trop d'interventions pour que ça me paraisse quelque chose de lisible, c'est vraiment un gribouillis.

MHPP: Pour vous.

PO: Pour moi, oui.

ZB: Je pense que pour les généticiens, plus il y a de gribouillis, mieux c'est ...

PO: J'ai beaucoup travaillé les cahiers de Paul Valéry. Je les ai travaillés bien sûr dans l'édition de la Pléiade que sa fille a faite, puis sur photos et maintenant il y a l'édition Gallimard qui est faite à partir des photos qu'on avait prises des manuscrits. J'apprécie beaucoup, moi aussi comme vous, le travail de généticiens du texte, sauf que je le fais moi de manière non méthodologique, c'est-à-dire que, ce qui m'intéresse, c'est de voir comment, par exemple, Valéry avait un imaginaire spatialisant. Il faisait bien souvent des dessins, il plaçait des textes à certains endroits de sa page en fonction des schémas qu'il faisait. Il faisait beaucoup de graphiques, et comme c'était quelqu'un hanté par la pensée mathématique, la pensée géométrique, je crois qu'il y a, effectivement, dans son écriture, une vision presque mathématicienne de la langue. Alors, là ça me paraît essentiel. Ce qui n'est pas mon cas parce que j'ai moins cet imaginaire graphique, disons plus oral.

MHPP: Votre processus est le même pour votre fiction et pour vos essais?

PO: Oui, assez oui, parce que j'accorde énormément d'importance au phrasé dans les essais, de plus en plus, parce que j'écris des essais un peu plus savants. Au tout début, j'étais plus influencé par les sciences humaines, par la sémiotique, par la linguistique, etc., donc c'était un langage un peu plus conceptuel jusqu'à Poétique $d u$ regard.

ZB: Maintenant c'est poétique.

AMLB: Vos essais sont poétiques.

PO: J'ai deux types d'essais. Les essais comme Où suis-je ou Hors temps ou Testaments, qui sont plus universitaires malgré tout, et puis des essais vraiment littéraires comme Asile, langue d'accueil, comme De $l^{\prime}$ Air. Ce sont des essais vraiment plus poétiques, il y en a un qui s'appelle La vie de mémoire. Un autre qui s'appelle Le premier venu.

ZB: Ah, j'adore La vie de Mémoire, c'est un peu le rapport avec votre père.

PO: C'est ça, exactement. 
ZB: Alors, pour finir, j'aurais une question sur vos impressions de cette expérience qu'on vient de vivre ensemble, de partager, de la Foire du livre de Porto Alegre.

PO: Ah, j'ai été très content de recevoir cette invitation parce que j'ai connu la Foire comme "spectateur", entre guillemets, une première fois quand j'étais venu il y a $7 \mathrm{ou}$ 8 ans je crois. J'avais été fasciné par cette Foire du Livre en plein air, parce que chez nous, les salons du livres on toujours lieu dans des endroits fermés, clos, un peu étouffants, et là justement, moi qui parle beaucoup d'air, je trouve particulièrement heureux que cela se fasse dans la rue, sur une place publique.

ZB: Avec les familles, les enfants...

PO: Avec les familles, tout ça... Alors, quand j'ai reçu l'invitation du bureau du Québec à São Paulo, j'ai tout de suite accepté, même si je suis un peu coincé parce que vous savez qu'à mon retour, il y a le salon du livre de Montréal où j'ai plusieurs activités autour de la revue les "Ecrits" dont on n'a pas pu parler mais qui est une activité très importante pour moi. Donc, je suis un peu coincé dans le temps mais je tenais a y être pour vivre un peu de l'intérieur ce qu'était cette foire, qui pour moi est unique au monde. On a un peu l'équivalent en minuscule, c'est le marché de la poésie à Montréal, qui est aussi sous la tente, sur une place publique, la place Gérald Godin sur le mont Royal.

ZB: Et à Paris aussi.

PO: Et à Paris aussi, place saint Sulpice. Donc, on a cette expérience un peu, mais ça n'a pas les dimensions de la Feira ici.

ZB: Il y a plus d'un million de visiteurs et presque 600 mille livres sont vendus.

PO: Moi, je rêve d'une ville librairie, une ville dont le centre serait entièrement habité par les livres et les œuvres d'art.

ZB: Oui, et ce n'est pas seulement les livres, c'est aussi la possibilité pour les gens, les lecteurs, de voir les écrivains qui vont parler, et il y a quantité de tables rondes, c'est incroyable, on risque de s'y perdre.

PO: Je vais vous donner un exemple pour revenir un peu à la première question que Zilá posait. Avanthier, je suis passé dans l'une des allées et j'ai entendu deux personnes, une comédienne et un musicien lire des poèmes de Hilda Hilst, donc en portugais, que moi j'ai lus plutôt en français car je ne connais pas assez bien le portugais. Je connais bien son œuvre mais je n'avais jamais entendu oralement ses textes. C'était magnifique, une comédienne accompagnée d'un guitariste qui faisait aussi une partie des voix. Donc, on se promène dans les allées et on entend des poèmes de Hilda Hilst, comme ça, récités et c'est formidable. On a ça un peu au marché de la poésie place saint Sulpice à Paris, que vous connaissez peut-être, où il y a aussi des poètes aux quatre coins qui lisent des textes pendant qu'on se promène dans les allées. Mais pas à la grandeur d'une ville, du centre ville.

ZB: Et pendant soixante ans sans interruption.

PO: Exactement. Et ça dure deux semaines, je crois?

ZB: Deux semaines. Cette année, dix-sept jours.

PO: Dix-sept jours, parce qu'à Paris, le marché de la poésie ne dure que quatre jours et à Montréal aussi ça ne dure que quatre jours seulement. J'adore ça. J'ai l'impression que la littérature a une présence dans cette ville, par ses libraires, par ses lecteurs, alors qu'on ne sent plus beaucoup ça dans les grandes villes d'Amérique du nord.

ZB: Nous en sommes très fiers.

Nous tenons à vous remercier vivement de votre disponibilité, de ce geste généreux de venir partager un peu de votre temps avec nous et nous permettre de combler quelques interstices qui se font lors de la lecture.

Recebido: 20 de novembro de 2014

Aprovado: 17 de dezembro de 2014

Contatos: ana.lisboa@pucrs.br marie-helene@pucrs.br zilabster@gmail.com 